\title{
The nanometer conductive film diffraction properties
}

\author{
VulfBorisovich Orlenson, Sergey Aleksandrovich Zuev, and Vladimir Victorovich Starostenko \\ V.I. Vernadsky Crimean Federal University, 295007, Simferopol, Russia
}

\begin{abstract}
In this article the numerical simulations and experimental studies of microwave electromagnetic wave interaction with micro- and nanoparticles of different sizes with their arbitrary location on the substrate are carried. The optical coefficients (reflection, transmission and absorption) and their dependence on the metallized nanocoating fill factor and thickness were obtained.
\end{abstract}

\section{Introduction}

Conductive films are an inalienable element of electronic devices and applications made using film technology. They are widely used in various fields, such as products and radio devices shielding from external electromagnetic field exposure, infrared sensing, and solar energy storage and in other branches of science and technology.

The properties of conductive films substantially depend on various parameters, in particular, on their thickness, which determines the mechanisms of electromagnetic field energy conversion into other types of energy. The main interaction mechanism of incident radiation with a conductive coating is ohmic losses, which convert the electromagnetic energy into thermal energy. The extension of thermoelectric processes in conductive films is strongly influenced by their spatial and ohmic heterogeneity, in particular this relates to contact pads, the burning of which is the main cause of integrated circuits (ICs) failures. In $[1,2]$ the film thickness dependences of the reflection coefficients for different conductive materials were deeply studied. Starting from a certain film thickness $(\sim 5-7 \mathrm{~nm})$, for most conductive materials, a rapid increase of the reflection coefficient $\mathrm{R}$ was observed. Such reflection behavior occurs due to the conductivity change and the formation of a reflective layer. The waveguide experimental studies in [3] give a more complete picture of the optical coefficients, where for an aluminum film thickness of $d=5 \mathrm{~nm}$ the absorption coefficient is $A \cong 25 \%$. Film thickness rising leads to a conductivity increase as a result the reflection grows while the absorption decreases. Also, the optical coefficients are frequency independent.

This paper presents the theoretical study of the conductive thin films absorbing properties behavior depending on metallized layer surface relief in the microwave range. Theoretical studies are carried out by solving the EM wave scattering problem through the numerical method of rigorous coupled wave analysis (RCWA) and analytical expressions of the Fresnel-Airy model [4].

*Corresponding author: starostenkovv@,cfuv.ru 


\section{Conductive thin film analysis}

In articles [5,6,7,8], experimental and theoretical studies of thin metal films absorbing properties were carried out in the long-wavelength part of IR spectrum and the microwave range. Irradiated by microwave EM waves conductive film of about 3 to $7 \mathrm{~nm}$ thickness range, exhibits quite significant absorbing properties. A further film thickness growth leads to increasing reflection. In particular interest are the [5.8] papers, in which the analytical expressions for conductive films optical coefficients were obtained in the considered range. These expressions have an interesting property, they are independent of the incident radiation frequency, and besides the absorption maximum of $50 \%$ is achieved under condition (1).

$$
\mathrm{R}_{\mathrm{s}}=\frac{1}{\sigma \mathrm{d}}=\frac{\mathrm{Z}_{0}}{2}=188,5 \Omega,
$$

where $R_{s}$ - conductive film sheet resistance, $\sigma$ - film conductivity $\mathrm{d}$ - film thickness and $\mathrm{Z}_{0}$ - free space impedance $\left(\mathrm{Z}_{0}=377 \Omega\right)$. The theoretical derivations described above were carried out under the assumption of conducting plane-parallel plate model with smooth boundaries. Maxwell's equations were solved in three layers and then stitched together at the boundaries between them. The first and the third layer describe the free space regions while the second layer between them describes the thin film region - the conductive layer. Such a problem statement can also be considered using the Fresnel - Airy model [4], the analytical expressions for which describe the complex reflection (2) and transmission (3) coefficients. В этой модели показатели преломления взяты такие же, как и в работах $[5,8]$. In this model, the refractive indices are taken the same as in $[5,8]$, so the optical coefficients are still frequency independent.

$$
\begin{aligned}
& r=\frac{r_{12}+r_{23} e^{-j 2 \psi}}{1+r_{12} r_{23} e^{-j 2 \psi}}, \\
& t=\frac{\left(1-r_{23}\right)\left(1-r_{12}\right) e^{-j \psi}}{1+r_{12} r_{23} e^{-j 2 \psi}}
\end{aligned}
$$

where $r_{12}, r_{23}$ - complex reflection coefficients between the layers 1 and 2, 2 and 3, with $\mathrm{R}=|\mathrm{r}|^{2}, \quad \mathrm{~T}=|\mathrm{t}|^{2} \quad-$ reflection and transmission coefficients, respectively, $A=1-\left(|r|^{2}+|t|^{2}\right)-$ absorption coefficients. At normal incidence $\psi=\mathrm{k}_{0} \tilde{\mathrm{n} d}$, where $\mathrm{k}_{0}-$ is wave vector at free space, $\mathrm{d}$ - conductive film thickness, $\tilde{\mathrm{n}}=\sqrt{\varepsilon_{\mathrm{r}} \mu_{\mathrm{r}}}-$ refractive index. The dielectric relative permittivity of conductive region is derived by $\varepsilon_{\mathrm{r}}=-\mathrm{j} \frac{\sigma}{\omega \varepsilon_{0}}$, where $\mathrm{j}=\sqrt{-1}, \omega-$ incident radiation angle frequency , $\varepsilon_{0}-$ absolute dielectric constant in vacuum and $\mu_{r}-$ magnetic relative permittivity $\left(\mu_{r}=1\right)$. In practice, the absorption maximum of a thin conductive (aluminum) film obtained by magnetron sputtering is observed at thicknesses from 5 to $10 \mathrm{~nm}[3,9]$. However, if the conductivity of crystalline aluminum equals to $\sigma_{\mathrm{c}-\mathrm{Al}}=3,8 \cdot 10^{7} \frac{\mathrm{s}}{\mathrm{m}}$ (or other crystalline metals with $\sigma$ of the order of $\sim 10^{7} \frac{\mathrm{s}}{\mathrm{m}}$ ) would be taken as the parameter $\sigma$, then the maximum absorption coefficient $\mathrm{A}=50 \%$ will be at $\mathrm{d} \approx 0,3 \mathrm{~nm}$ thickness [10,11], which does not coincide with the experimental data. Using crystalline conductivity is not reasonably, since as a result of sputtering techniques an amorphous conductive material is deposited on the substrate surface. According to condition (1), the film conductivity for the considered thickness should be of the order of $\sim 10^{6} \frac{\mathrm{s}}{\mathrm{m}}$, which may well correspond to the conductivity of amorphous aluminum. 


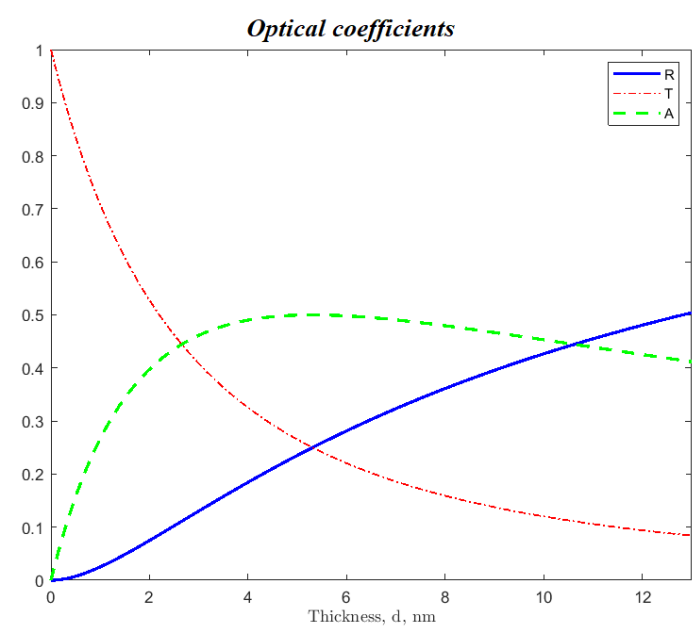

Fig. 1. Optical coefficients dependence on the film thickness obtained by 1D model simulation.

\section{Diffraction properties theoretical studies of conductive nanofilms}

The properties of conductive films are associated not only with their thickness, but also with the physical processes, which are inextricable linked with the characteristics of the substrates on which they are deposited, forming metal-dielectric structures (MDS). The onedimensional formulation of EM wave scattering problem made it possible to obtain the condition of reaching the absorption maximum (1). However, the real MDSs surface topography studies by atomic and tunneling microscopy showed that such a problem statement does not allow spatial and ohmic inhomogeneities analysis of the conductive layer in terms of their contribution to the metal film absorbing properties. To solve this problem, the MDS optical characteristics should be carried out using a 3D electromagnetic film model.

To calculate the optical coefficients, the numerical method of rigorous coupled wave analysis (RCWA) was applied [12]. This method solves Maxwell's equations in Fourierspace. To study multilayer optical systems with complex relief, in the transverse direction of each layer the eigenvalue problem is solved, after which the results are stitched together at the boundaries of all layers. The $2 \mathrm{D}$ dielectric constant function of each layer should be represented by a high-resolution real-space discrete grid, and then transformed in reciprocal space (Fourier-space) (4).

$$
\varepsilon\left(\mathrm{k}_{\mathrm{x}}(\mathrm{p}), \mathrm{k}_{\mathrm{y}}(\mathrm{q})\right)=\frac{1}{\mathrm{~N}_{\mathrm{x}} \cdot \mathrm{N}_{\mathrm{y}}} \sum_{\mathrm{i}=1}^{\mathrm{N}_{\mathrm{x}}} \sum_{\mathrm{k}=1}^{\mathrm{N}_{\mathrm{y}}} \varepsilon\left(\mathrm{x}_{\mathrm{i}}, \mathrm{y}_{\mathrm{k}}\right) \mathrm{e}^{-\mathrm{j} \cdot\left(\mathrm{k}_{\mathrm{x}}(\mathrm{p}) \cdot \mathrm{x}_{\mathrm{i}}+\mathrm{k}_{\mathrm{y}}(\mathrm{q}) \cdot \mathrm{y}_{\mathrm{k}}\right)},
$$

where $\mathrm{k}_{\mathrm{x}}(\mathrm{p}), \mathrm{k}_{\mathrm{y}}(\mathrm{q})$ - transverse wave vector components, $\mathrm{p}, \mathrm{q}$ - spatial harmonics along $x$ and $y$ axes respectively, $\mathrm{N}_{\mathrm{x}}, \mathrm{N}_{\mathrm{y}}-x-y$ grid discrete points.

In this work, the S-matrices [13] method is used to carry out the procedure of solution stitching and optical coefficients calculating. To calculate the optical coefficients, all the considered energy contributions of the reflected and transmitted spatial harmonics should be summed (5), (6).

$$
\mathrm{T}=\sum_{\mathrm{p}=1}^{\mathrm{P}} \sum_{\mathrm{q}=1}^{\mathrm{Q}} \mathrm{T}(\mathrm{p}, \mathrm{q}), \mathrm{R}=\sum_{\mathrm{p}=1}^{\mathrm{R}+\mathrm{T}+\mathrm{A}=1} \sum_{\mathrm{q}=1}^{\mathrm{Q}} \mathrm{R}(\mathrm{p}, \mathrm{q})
$$


where $\mathbf{R}$ - reflection coefficient, $\mathrm{T}$ - transmission coefficient и $\mathrm{A}$ - absorption coefficient.

The 3D model of the conductive film deposited on the substrate surface is presented in layered 2D periodic lattice form with $\Lambda_{\mathrm{x}}$ and $\Lambda_{\mathrm{y}}$ periods along the corresponding axes $\mathrm{x}$ and $y$. The unit cell of the conducting film is described by the $2 \mathrm{D}$ function $\varepsilon_{\mathrm{r}}(\mathrm{x}, \mathrm{y})$, the real part of which is shown in fig. $2 \mathrm{a}, 3 \mathrm{a}$. The incident EM wave is linearly polarized, and its electric field vector is directed along the larger diagonal of the elliptical conducting particle. For the same reasons as in the case of a 1D model, the conductivity of a metal particle is $\sigma=10^{6} \frac{\mathrm{s}}{\mathrm{m}}$. The substrate layer thickness is $\mathrm{d}_{\text {sub }}=12 \mu \mathrm{m}$, with a relative dielectric constant $\varepsilon_{\mathrm{r}_{\text {sub }}}=10$. Based on fig. 1 at a thickness $\mathrm{d}=4 \mathrm{~nm}$, in which the maximum absorption $A=50 \%$ should be achieved the influence of conductive layer geometry on the optical characteristics can be studied. In the RCWA it is very important to look for convergence, since the conservation of power will always be obeyed even considering just one spatial harmonic, therefore, to obtain correct results, it is necessary to take into account such number of spatial harmonics at which the changes in optical coefficients will be insignificant. To solve the current problem the $\mathrm{N}_{\mathrm{x}}=\mathrm{N}_{\mathrm{y}}=512$ real-space grid resolution was chosen.

Fig. 2 shows optical coefficients plots for an array of periodically located elliptical conducting particles. Such an MDS does not have continuous conduction channels; therefore, taking into account a greater number of spatial harmonics the transmission coefficient $T \rightarrow 1$, fig. $2 \mathrm{~b}$. In this case, the convergence of the results is achieved with a large number of spatial harmonics.

a)

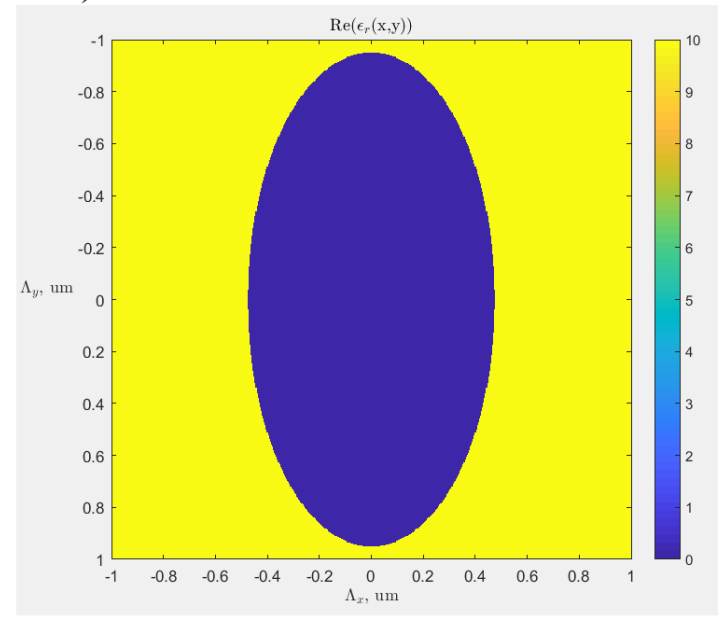

b)

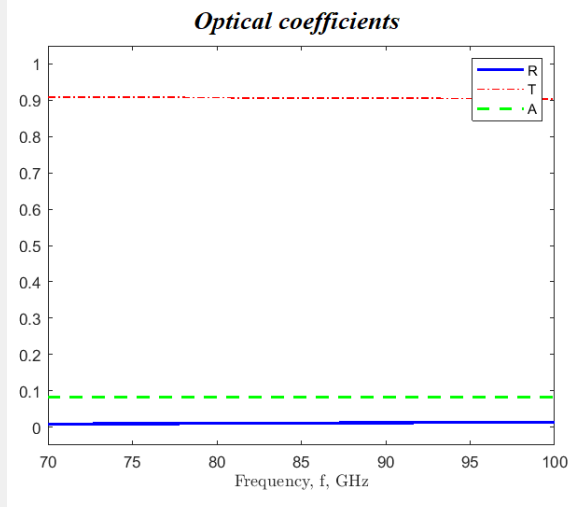

Fig. 2. Metallized surface consisting of periodically arranged elliptical conductive particles: $\mathrm{a}$ - unit cell dielectric constant plot, $\mathrm{b}$ - optical coefficients plot.

An increase elliptical particle size till it crosses the unit cell boundary leads to the formation of continuous conducting channels, fig. $3 \mathrm{a}$. In this case, the transmission coefficient decreases to about $65 \%$ while the absorption coefficient increases to $30 \%$, fig.3b. For such an MDS, the results converge with a much smaller number of spatial harmonics, $P=Q=23$.

Based on the obtained calculations, it can be concluded that the absorbing properties of a metal nanofilm strongly depend on the spatial distribution of its conductive particles, although their linear dimensions and the distance between them are much smaller than the incident wavelength, fig.4. The absorption maximum is achieved only with the formation of continuous conductive channels. 
a)

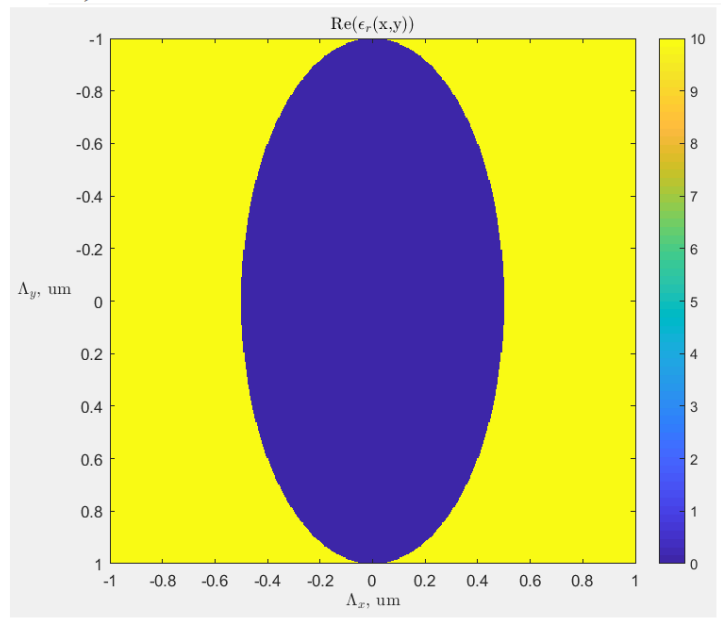

b)

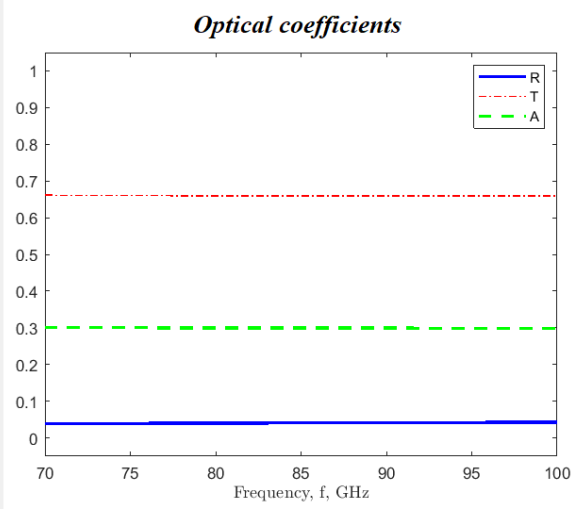

Fig. 3. Metallized surface consisting of periodically arranged intersecting elliptical conductive particles: $\mathrm{a}$ - unit cell dielectric constant plot, $\mathrm{b}$ - optical coefficients plot.
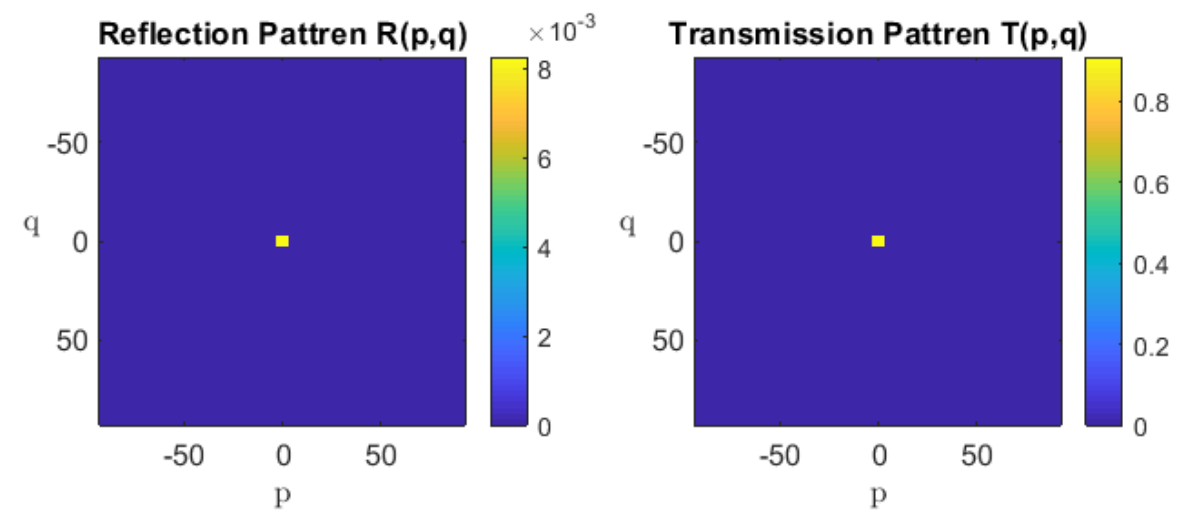

Fig. 4. Spatial harmonics power composition studies. Transmission and reflection coefficient dependence on spatial harmonics. The main energy of microwave radiation is contained only in the main spatial harmonic.

\section{Conclusion}

The two description approaches of optical coefficients calculation are presented for metaldielectric structures with aluminum nanofilms. Numerical calculations show a strong dependence of microwave range optical coefficients behavior on the conductivity, the thickness and the spatial distribution of the conductive material on the substrate surface. This type of EM wave scattering is realized due to structure transition from dielectric (the film is absent) to a conducting film that short-circuited the space.

\section{References}

1. I.V. Antonetz, L.N. Kotov, S.V. Nekipelov, S.V. Karpushev. Technical Physics 74(11) p. 102-106 (2004)

2. I.V. Antonetz, L.N. Kotov, E.A. Golubev, S.N. Petrunev. Arctic Evironmental Research p. 50-57, (2008) 
3. S.P. Arsenichev, M.V. Glumova, E.V. Grigoryev, S.A. Zuev, A.S. Mazinov, V.B. Orlenson, A.G. Nalogin, V.V.Starostenko, I.Sh. Fitaev. Surface characteristics of amorphous substrates and nanometer conductive films. CriMiCo'2018 28th International Crimean Conference Microwave and Telecommunication Technology. Conference Proceedings. Sevastopol, 1022 (2018)

4. R. C. Rumpf http://emlab.utep.edu, Multiple Scattering.

5. W. Woltersdorff The Optical Constants of Thin Metal Films in the Long-Wave UltraRed Region. Z. Physilc, 91, 230 (1934)

6. H. Murmann, Z. Phyzik, 54, 741 (1929)

7. M. Cherny, H. Reder. Infrared Technology Advances. Physics-Uspekhi 25(1) (1941)

8. Sucheng Li, Shahzad Anwar, Weixin Lu, Zhi Hong Hang, Bo Hou, Mingrong Shen, and Chin-Hua Wang. Microwave absorptions of ultrathin conductive films and designs of frequency-independent ultrathin absorbers. AIP ADVANCES, 4 (2014)

9. V.G. Andreev, V.A. Vdovin, S.M. Pronin, I.A. Horin. Measurement of nanometer metal films optical coefficients at a frequency of $10 \mathrm{GHz}$. Journal of Radio Electronics ISSN 1684-1719, 11 (2017)

10. V.A. Vdovin. Nanometer metal films in sensors of powerful microwave pulses. III Russian Conference «Radar and radio communications» - Kotel'nikov institute of radio engineering and electronics of RAS, 26 - 30 october (2009)

11. S.M. Pronin, V.A. Vdovin, V.G. Andreev. Optical coefficients investigation of copper and gold nanometer films in the microwave range. Physics Faculty scientific letters. 5 (2016)

12. M. G. Moharam, Drew A. Pommet, Eric B. Grann, "Stable implementation of the rigorous coupled-wave analysis for surface-relief gratings: enhanced transmittance matrix approach. J. Opt. Soc. Am. A, Vol. 12, No. 5, pp. 1077-1086 (May 1995)

13. R.C. Rumpf. Improved formulation of scattering matrices for semi-analytical method that is consistent with convention. Progress In Electromagnetics Research B, Vol. 35, $241-261(2011)$ 